\title{
DISCOVERY OF BLUE HOOK STARS IN THE MASSIVE GLOBULAR CLUSTER M54
}

\author{
Alfred Rosenberg \\ Instituto de Astrofísica de Canarias, Vía Láctea s/n, E-38200 La Laguna, Tenerife, Spain; alf@11.iac.es \\ Alejandra Recio-Blanco \\ Dipartimento di Astronomia, Università di Padova, Vicolo dell’Osservatorio 2, I-35122 Padua, Italy; recio@pd.astro.it \\ AND \\ Macarena García-Marín \\ Departamento de Astrofísica Molecular e Infrarroja, Instituto de Estructura de la Materia, CSIC, \\ Serrano 113 bis, E-28006 Madrid, Spain; maca@damir.iem.csic.es \\ Received 2003 September 12; accepted 2003 November 12
}

\begin{abstract}
We present $B V$ photometry centered on the globular cluster M54 (NGC 6715). The color-magnitude diagram clearly shows a blue horizontal branch extending anomalously beyond the zero-age horizontal-branch theoretical models. These kinds of horizontal-branch stars (also called "blue hook" stars), which go beyond the lower limit of the envelope mass of canonical horizontal-branch hot stars, have so far been known to exist in only a few globular clusters: NGC 2808, $\omega$ Centauri (NGC 5139), NGC 6273, and NGC 6388. Those clusters, like M54, are among the most luminous in our Galaxy, indicating a possible correlation between the existence of these types of horizontal-branch stars and the total mass of the cluster. A gap in the observed horizontal branch of M54 around $T_{\text {eff }}=27,000 \mathrm{~K}$ could be interpreted within the late helium flash theoretical scenario, which is a possible explanation for the origin of blue hook stars.
\end{abstract}

Subject headings: globular clusters: individual (NGC 6715) - Hertzsprung-Russell diagram — stars: horizontal-branch

\section{INTRODUCTION}

The horizontal branch (HB) hosts stars with a heliumburning core of about $0.5 M_{\odot}$ and a hydrogen-burning shell. The masses of the hydrogen envelopes vary from more than $0.2 M_{\odot}$ to less than $0.02 M_{\odot}$. Furthermore, the less massive the hydrogen envelope is, the hotter is the corresponding HB star. In the case of a star cluster, we find a color spread of the HB stars which is called the HB morphology. To a first approximation, the different color extensions of observed cluster HBs are described in terms of the variation of metal abundance, which is taken as the first parameter (metal-rich clusters tend to have short red HBs, while metal-poor ones exhibit predominantly blue HBs). However, some other parameter or set of parameters also has to be at work, as clusters with nearly identical metallicities can show very different HB color distributions (van den Bergh 1967; Sandage \& Wildey 1967), leading to the so-called second-parameter debate.

Horizontal branch stars with very low envelope masses $\left(\leq 0.02 M_{\odot}, T_{\text {eff }}>20,000 \mathrm{~K}\right)$, known as extended or "extreme HB" (EHB) stars, are probably the most extreme expression of the second-parameter problem. They have lost up to twice as much mass during the red giant branch (RGB) ascent as other HB stars in the same cluster (D'Cruz et al. 1996). As a result, in contrast to the more massive blue HB stars, EHB stars do not ascend the asymptotic giant branch (AGB), but evolve directly into the white dwarf domain (Sweigart, Mengel, \& Demarque 1974). Recently, Whitney et al. (1998) and D'Cruz et al. (2000) revealed the existence of a particular kind of EHB star: a population of hot subluminous HB stars, lying up to 0.7 mag below the zero-age horizontal branch (ZAHB) and forming a hooklike feature in the far-UV color-magnitude diagram (CMD) of $\omega$ Centauri. These "blue hook" stars have effective temperatures up to $40,000 \mathrm{~K}$ and cannot be produced by canonical HB evolution (Brown et al. 2001). In the optical, for effective temperatures higher than $10,000 \mathrm{~K}$, ultraviolet radiation constitutes the main part of the energy flux coming from the stellar surface, making the HB, in practice, vertical in the classical $V$ versus $(B-V)$ plane because of bolometric correction. Hence, in optical CMDs, blue hook stars are located at the faintest extreme of the HB.

In this paper, we present $B V$ photometry centered on the globular cluster (GC) M54. The CMD clearly shows a blue HB anomalously extending beyond ZAHB models. Previous photometric studies of this cluster in $(V-I)$ were not suitable for properly revealing this extremely hot stellar population. Initially, blue hook stars were detected in the clusters NGC 2808 and $\omega$ Centauri. More recently, Busso, Piotto, \& Cassisi (2004) have reported their presence also in the blue HB tail of NGC 6388. In addition, as noted by Brown et al. (2001), the CMD of NGC 6273 given by Piotto et al. (1999) shows a blue HB extending to $M_{V}>5$ and therefore beyond theoretical ZAHB models. All these clusters are among the most massive GCs in the Galaxy, as is M54, which is the second most massive GC known.

In $\S 2$, we describe the observations and the photometric reduction techniques. In $\S 3$, we analyze the extended HB of M54 and its interpretation inside the late helium flashers scenario. Finally, in $\S 4$, we summarize the results and consider their wider implications.

\section{OBSERVATIONS AND REDUCTION}

The observational database consists of four images, two in $B$ and two in $V$, of 30 and $900 \mathrm{~s}$ each, centered on M54. Images were observed in service mode on 2002 July 8 at the ESO $3.5 \mathrm{~m}$ New Technology Telescope (NTT), with a seeing of $\sim 0$ " 5 . The detector, the Superb Seeing Imager (SUSI2), is a mosaic of 
two $2 \mathrm{~K} \times 4 \mathrm{~K}$ EEV CCDs, with a size of $0^{\prime \prime} 08$ pixel $^{-1}$ and a total field of view of $5.5 \times 5.5$ that were binned $2 \times 2$.

The images were corrected for bias and spatial sensitivity variations using the respective master flats, which were computed as the median of all available sky flats of the specific run. Afterward, photometry was performed using the DAOPHOT/ALLSTAR/ALLFRAME software (Stetson 1987, 1994).

The absolute calibration of the observations, which include the BVRI filters, will be published in a forthcoming paper. It is based on six fields of standard stars from the catalog of Landolt (1992), and the absolute zero-point uncertainties of our calibration are $\leq 0.02$ mag for each of the four bands.

\section{THE EXTENDED HB OF M54 AND ITS INTERPRETATION}

Figure 1 shows the CMD of M54 in an area away from the crowded region with $R<90^{\prime \prime}$, where $R$ is the projected distance from the cluster center. We can identify at least three stellar systems: the GC M54, the Sagittarius dwarf galaxy, and the Milky Way bulge (Layden \& Sarajedini 2000). All stars plotted were selected using the sharp parameter $(-0.25<$ $s h<0.25)$ and the $V$ error $(\leq 0.1)$. In this paper we are interested in M54, in which the main sequence (MS), RGB, and HB are clearly visible. The whole CMD has been shifted in color and magnitude by $E(B-V)=0.16$ and $(m-M)_{0}=$ 17.25 in order to fit the ZAHB model. The CMD shows an extended HB that spans almost 4.75 mag in $V$ and extends down to $M_{V} \simeq 5.25$, i.e., $\sim 1.5 \mathrm{mag}$ below the turnoff. The thick line shows the ZAHB model by Cassisi \& Salaris (1997) for a metallicity of $[\mathrm{Fe} / \mathrm{H}]=-1.31$.

Crowding and completeness experiments were also performed along the HB sequence. The completeness was found to be higher than $50 \%$ for the entire CMD shown $(60 \%$ and $70 \%$ zones are labeled in Fig. 1).

From the crowding experiments, we have obtained the photometric dispersion for synthetic stars located on the HB theoretical model line, to which we have added 3 times the typical dispersion in color $(0.025 \mathrm{mag})$ measured from the region between $1.0 \leq M_{V} \leq 4.5$ of the HB. These limits are represented by the dashed lines, and all 78 stars within these two lines are considered HB stars in the following discussion.

Although, because of bolometric correction, the $(B-V)$ color is not a good temperature indicator for the hot HB stars, the CMD clearly shows a horizontal branch $\sim 0.5 \mathrm{mag}$ deeper than the ZAHB model. At least nine stars in Figure 1 seem to have effective temperatures higher than $33,600 \mathrm{~K}$, which is the end of the ZAHB model by Cassisi \& Salaris (1997). As mentioned in $\S 1$, a different evolutionary path for populating the very hot end of the HB is therefore needed. One possible explanation is that these stars experience a delayed helium core flash. Castellani \& Castellani (1993) and D'Cruz et al. $(1996,2000)$ showed that even if a star suffers a very high mass loss during its RGB phase, it can still ignite helium burning after evolving off the RGB.

If the helium flash occurs somewhere between the tip of the RGB and the top of the white dwarf cooling curve (early hot flashers), the star will settle onto the EHB without any mixing between its helium core and hydrogen envelope, following a canonical evolutionary path to the EHB. The reason for this can be found in the entropy barrier caused by the hydrogen-

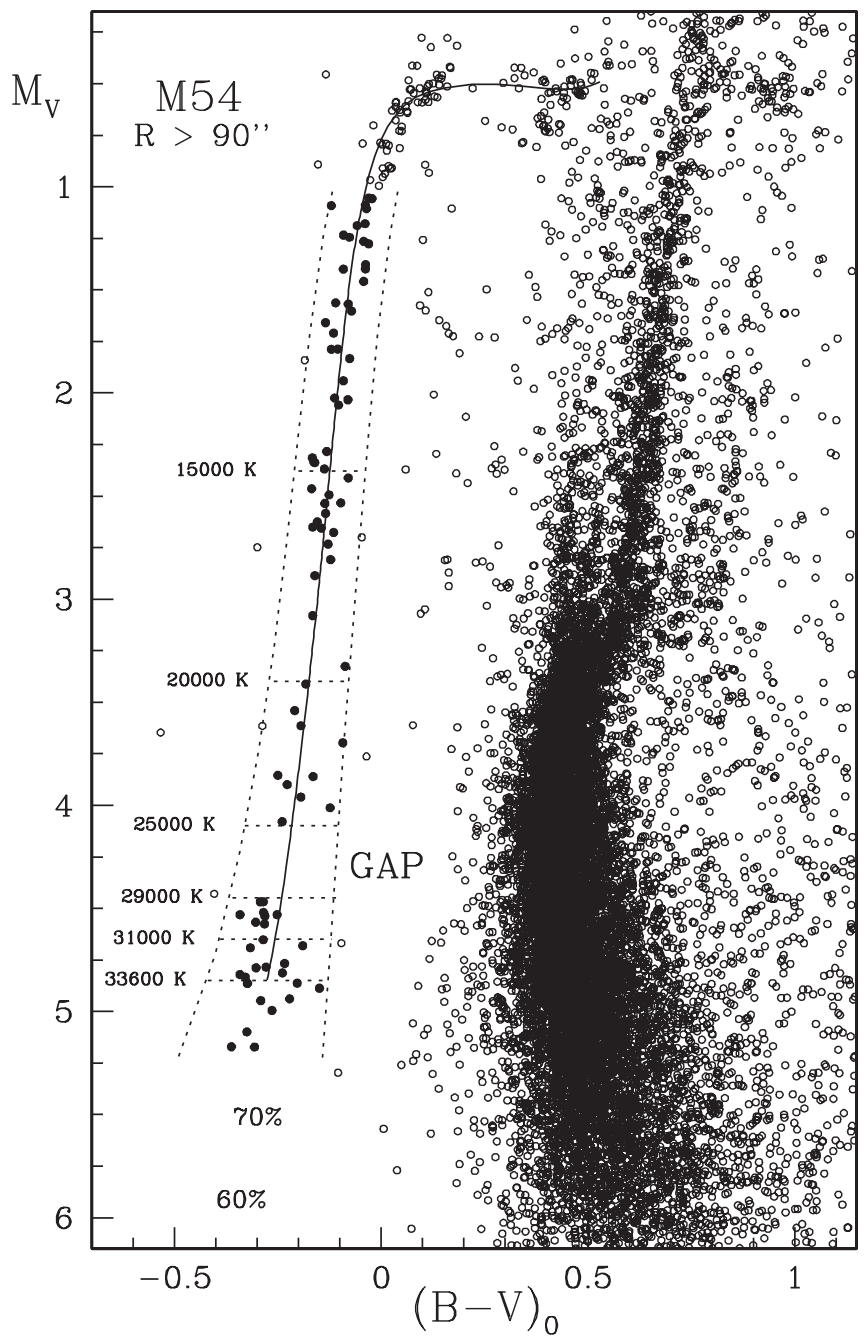

FIG. 1. $-M_{V}$ vs. $(B-V)_{0}$ CMD for 15,202 stars from the observed field centered on M54. All stars plotted were selected by the sharp parameter $(-0.25<s h<0.25)$ and the $V$ error $(<0.1)$, and are all farther than $90^{\prime \prime}$ from the cluster center. The overplotted thick line is the ZAHB model by Cassisi \& Salaris (1997), for a metallicity of $[\mathrm{Fe} / \mathrm{H}]=-1.31$. On both sides, dashed lines show the color limits of the region within which we have adopted stars as HB stars. Several temperatures obtained from the ZAHB model are also shown. Note the gap between 25,000 and 29,000 K. The 27 stars below the gap and between the dashed lines are expected to be blue hook stars. The $70 \%$ and $60 \%$ completeness levels, obtained from a crowding experiment along the HB region, are also labeled.

burning shell, which prevents the convection zone produced by the helium flash from penetrating into the hydrogen envelope (Iben 1976). Early hot flasher models by Brown et al. (2001) predict that these stars reach a maximum temperature of $31,500 \mathrm{~K}$ on the EHB and that their luminosities are almost indistinguishable from the luminosities of canonical EHB stars. Brown et al. (2001) claimed that if the helium flash occurs while the star is descending the white dwarf cooling curve (late hot flashers), the entropy barrier carried by the hydrogen-burning shell becomes too weak to prevent mixing between the core and the star's envelope. The resulting star will have a temperature of about $37,000 \mathrm{~K}$ and will lie as much as 0.7 mag below the ZAHB in the UV CMD, further supporting the argument that blue hook stars are the progeny of late hot flashers. The fact that blue hook stars are both hotter and more helium-rich than classical EHB stars has been observationally confirmed by the spectroscopic analysis 
of Moehler et al. (2002) in $\omega$ Centauri. In addition, full evolutionary computation of helium flash-induced mixing in Population II stars has recently been developed by Cassisi et al. (2003). They modeled the incursion of the helium flashdriven convective zone into the hydrogen-rich external layers with a subsequent surface enrichment in helium and carbon. In agreement with the observations, models experiencing this dredge-up event are significantly hotter than their counterparts with hydrogen-rich envelopes. They also compare their abundance predictions of helium with measurements by Moehler et al. (2002).

On the other hand, the sharp transition between the early and late hot flashers, corresponding to a difference in mass loss of only $10^{-4} M_{\odot}$ along the RGB, would produce a gap in the observed stellar distribution. The CMDs of $\omega$ Centauri by Kaluzny et al. (1997) and Lee et al. (1999) and NGC 6273 by Piotto et al. (1999) do not seem to show an EHB gap. Brown et al. (2001) suggest that the gap in $\omega$ Centauri is perhaps blurred by the metallicity distribution of the cluster. The differential reddening and magnitude limit could be the reason in the case of NGC 6273. Brown et al. (2001) test their blue hook models by trying to reproduce the luminosity function of NGC 2808 by Bedin et al. (2000). They first assume that the EHB and the blue hook zone are uniformly populated and then include the HB evolution of the models to brighter $V$ magnitudes. They find that the gap in the observed ZAHB distribution corresponds to the gap between the canonical ZAHB and their blue hook models with mixed envelopes, further supporting the scenario of the late hot flashers.

In Figure 2, we compare the stellar distribution in $M_{V}$ of the M54 HB from our photometry (filled triangles) with that of NGC 2808 by Bedin et al. (2000) (open circles). The total number of stars in the NGC 2808 distribution (160 stars) was normalized to that of M54 (78 stars) for a better comparison. For the latter cluster, a distance modulus of $(M-m)_{V}=15.74$ (Bedin et al. 2000) was used. Error bars for M54 correspond to the square root of the number of the stars per bin, and the bin size is $0.30 \mathrm{mag}$. Interestingly, M54 presents a gap in the stellar distribution at the same absolute $V$ magnitude $\left(M_{V} \sim 4.3\right)$ as NGC 2808 (marked in Fig. 2 with a vertical arrow). As pointed out before, in the late helium flasher scenario, this gap would separate the canonical EHB from the blue hook stars and would be only apparent because of the mixed envelopes of blue hook stars (Brown et al. 2001). If this is true, a quite abundant blue hook population would be clearly seen in M54 at the same absolute magnitudes

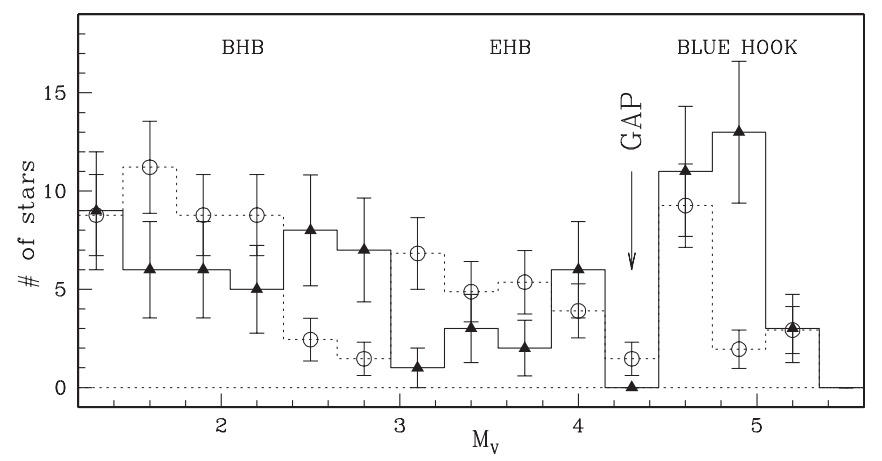

FIG. 2.-Stellar distribution of the M54 HB (filled triangles) from our photometry and NGC 2808 (open circles) from Bedin et al. (2000). The same gap at $M_{V} \sim 4.3$ is observed, possibly separating the canonical HB from blue hook stars. (effective temperatures) as in NGC 2808. From this histogram, we found that the number of stars in the blue hook region compared to the whole HB star sample (for $1.0 \leq M_{V} \leq 5.5$ ) is $35 \%$ in the case of M54 and less than $20 \%$ in the case of NGC 2808. We do not know if this difference is caused by the metallicities, which are very similar for both clusters $([\mathrm{Fe} / \mathrm{H}]=-1.25$ and $[\mathrm{Fe} / \mathrm{H}]=-1.11$ for M54 and NGC 2808, respectively, in the Carretta \& Gratton [1997] scale [Rutledge, Hesser, \& Stetson 1997]), which helps the direct comparison of their HBs. On the other hand, it would be necessary to take into account the HB evolution of the models to brighter $V$ luminosities in order to explain the location of the gap at $T_{\text {eff }} \sim 27,000 \mathrm{~K}$, as already claimed by Brown et al. (2001) for the case of NGC 2808. However, we note that the first gap in the HB of NGC 2808 pointed out by Bedin et al. (2000) at $V \sim 18.5 \mathrm{mag}\left(M_{V} \sim 2.8 \mathrm{mag}\right)$ is not visible in M54, although there is an underpopulated zone at lower magnitudes around $M_{V} \sim 3.2 \mathrm{mag}$.

Finally, the peculiar bimodality of the NGC 2808 HB morphology, which includes both a blue HB and a red HB clump, may also be present in M54. This would increase the similarities in the peculiar features observed in the HB of these two clusters. However, although there is evidence for a red HB in both our diagram and already published VI photometry (see, for example, Sarajedini \& Layden 1995), this feature could belong to the Sagittarius (Sgr) dwarf galaxy field. Layden \& Sarajedini (2000), in their extensive VI photometry of M54 and the Sgr galaxy, conclude that their subtraction of the Sgr field from the M54 CMD cannot ascertain beyond doubt whether the anonymous red HB belongs to Sgr or to M54. On the other hand, in the recent work of Monaco et al. (2003), it is clear that the Sgr dwarf galaxy also possesses an old and relatively metal-poor stellar component that populates the blue HB tail. Interestingly, Monaco et al. (2003) find that the faintest $(V>18.6)$ blue HB stars are relatively less populous in the Sgr field than in M54. Moreover, the spread of the stars in the hottest part of the Sgr field HB is rather large. In our Figure 1 we see that the stars that we present as blue hook stars are clearly grouped in a relatively small region of the CMD. They are clearly separate from the MS, since the rest of our CMD is extremely clean. For all these reasons, we believe that the stars in our CMD located under the gap are indeed cluster members.

\section{DISCUSSION}

As described in the previous section, we conclude that the M54 HB hosts a blue hook stellar population that extends the HB to fainter magnitudes than ZAHB models. Understanding the origin of hot HB stars not only is important for our finetuning of stellar evolution theory, but also has wider applications in astrophysics. Indeed, hot HB stars are now considered to be the prime contributors to the ultraviolet emission in elliptical galaxies (Greggio \& Renzini 1990; Brown et al. 2001). Blue hook stars are not, however, a common feature of all GCs with an extended blue HB morphology (see, for instance, Moehler et al. 2000 for the cluster NGC 6752).

At this point, it might be useful to examine the similarities in the physical properties of this cluster with the other GCs with confirmed or suspected blue hook stars in their HBsthat is, NGC 2808, NGC 6388, NGC 6273, and $\omega$ Centauriin order to shed more light on the origin of this peculiar kind of star. The most striking similarity between these five clusters is that they are among the most massive clusters in our Galaxy. 
This characteristic would explain the presence of a larger EHB population, but would not, in principle, be directly considered as a justification for the bluer HB morphology of these clusters. The total absolute $V$ magnitude $\left(M_{V}\right)$ can give a good estimate of these clusters' total luminosities (as all GCs have similar color indices and hence similar bolometric corrections) and therefore a good measurement of the baryonic mass of these old stellar systems. From Harris (1996; updated to the new catalog version of 2003 February), we find $M_{V}=-9.18$ for NGC $6273,-9.39$ for NGC $2808,-9.42$ for NGC 6388, -10.01 for M54, and -10.29 for $\omega$ Centauri.

Nevertheless, we might think that if there is a distribution in mass loss along the RGB, then the high mass loss tail of this distribution would be more likely to be occupied in a more massive cluster. If this were true, a correlation between the number of hot HB stars per stellar mass in a cluster and its HB extension and/or total mass at constant metallicity should also exist. The first results of a multivariate analysis (A. RecioBlanco et al. 2004, in preparation) based on a photometric database of 74 GCs (Piotto et al. 2002) seem to exclude such a correlation.

On the other hand, both M54 and $\omega$ Centauri are suspected of being the nuclei of a current and former dwarf galaxy, respectively. In fact, M54 must have played an important role in the star formation history of the Sagittarius galaxy, as it lies in the high-density region of Sgr (Ibata et al. 1997), and, as pointed out by Layden \& Sarajedini (2000), it marks one of the earliest epochs of star formation in Sgr. M54 may be similar to the nuclear star clusters seen in nucleated dwarf elliptical galaxies (Sarajedini \& Layden 1995). On the other hand, because of the unusual properties of $\omega$ Centauri (mainly abundance variations and metallicity spread), the scenario that this cluster may also be the core of a disrupted dwarf galaxy (e.g., Freeman 1993) that had a continuous infalling of gas to its center, leading to a variable star formation history, is becoming popular. Moreover, the NGC 2808 HB bimodality could be interpreted within a similar scenario of cluster self- enrichment if we consider the D'Antona et al. (2002) suggestion of the influence of a possible second generation of helium-rich stars in the final cluster HB morphology. In this sense, the correlation between the high mass of these clusters and the existence of blue hook stars (and so of their progenitors as the proposed late hot helium flashers) could also be linked to the second-parameter debate regarding the more general problem of GC HB morphology.

It is interesting to point out, in addition, that age differences from cluster to cluster would not be enough to explain the second-parameter problem. This is the case for NGC 2808, coeval with other clusters of similar metallicity but much shorter HBs such as NGC 362, NGC 1261, or NGC 1851, all of which are still $20 \%$ younger than NGC 288, NGC 5904, or NGC 6218 (Rosenberg et al. 1999, 2000a, 2000b). In fact, many other massive GCs of different metallicities show particularly extended HB morphologies: NGC $6266\left(M_{V}=-9.19\right.$, $[\mathrm{Fe} / \mathrm{H}] \sim-1.3), \operatorname{NGC} 2419\left(M_{V}=-9.58,[\mathrm{Fe} / \mathrm{H}] \sim-2.1\right)$ and NGC $6441\left(M_{V}=-9.64,[\mathrm{Fe} / \mathrm{H}] \sim-0.5\right)$. Deeper photometry in the blue or in the ultraviolet could reveal the presence of a blue hook population for those clusters like the ones already detected in NGC 2808, $\omega$ Centauri, NGC 6388, NGC 6273, and now in M54.

We would like to thank our anonymous referee for many valuable comments and suggestions. This report is based on observations with the ESO NTT and SUSI2, located at the La Silla Observatory, Chile [ESO proposal 69.D-0655(A)]. We thank L. R. ("Rolly") Bedin for providing the NGC 2808 HB photometry and Santino Cassisi for his ZAHB models. We are grateful to Antonio Aparicio, Carme Gallart, Giampaolo Piotto, and Ivo Saviane for allowing us to use their data from a larger project entitled "Relative Ages of Outer Halo Globular Clusters" in advance of publication. A. R.-B. recognizes support from MIUR and from ASI.
Bedin, L. R., Piotto, G., Zoccali, M., Stetson, P. B., Saviane, I., Cassisi, S., \& Bono, G. 2000, A\&A, 363, 159

Brown, T. M., Sweigart, A. V., Lanz, T., Landsman, W. B., \& Hubeny, I. 2001, ApJ, 562, 368

Busso, G., Piotto, G., \& Cassisi, S. 2004, Mem. Soc. Astron. Italiana, 75, 46

Carretta, E., \& Gratton, R. G. 1997, A\&AS, 121, 95

Cassisi, S., \& Salaris, M. 1997, MNRAS, 285, 593

Cassisi, S., Schlattl, H., Salaris, M., \& Weiss, A. 2003, ApJ, 582, L43

Castellani, M., \& Castellani, V. 1993, ApJ, 407, 649

D’Antona, F., Caloi, V., Montalbán, J., Ventura, P., \& Gratton, R. 2002, A\&A, 395, 69

D’Cruz, N. L., Dorman, B., Rood, R. T., \& O’Connell, R. W. 1996, ApJ, 466,359

D'Cruz, N. L., et al. 2000, ApJ, 530, 352

Freeman, K. C. 1993, in ASP Conf. Ser. 48, The Globular Cluster-Galaxy Connection, ed. G. H. Smith \& J. P. Brodie (San Francisco: ASP), 27

Greggio, L., \& Renzini, A. 1990, ApJ, 364, 35

Harris, W. 1996, AJ, 112, 1487

Ibata, R. A., Wyse, R. F., Gilmore, G., Irwin, M. J., \& Suntzeff, N. B. 1997, AJ, 113,634

Iben, I. 1976, ApJ, 208, 165

Kaluzny, J., Kubiak, M., Szymański, M., Udalski, A., Krsemiński, W., \& Mateo, M. 1997, A\&AS, 125, 343

\section{Landolt, A. U. 1992, AJ, 104, 340}

Layden, A. C., \& Sarajedini, A. 2000, AJ, 119, 1760

Lee, Y. W., Joo, J. M., Sohn, Y. J., Rey, S. C., Lee, H. C., \& Walker, A. R. 1999, Nature, 402, 55

Moehler, S., Sweigart, A. V., Landsman, W. B., \& Driezler, S. 2002, A\&A, 395, 37

Moehler, S., Sweigart, A. V., Landsman, W. B., \& Heber, U. 2000, A\&A, 360,120

Monaco, L., Bellazzini, M., Ferraro, F. R., \& Pancino, E. 2003, ApJ, 597, L25

Piotto, G., Zoccali, M., King, I. R., Djorgovski, S. G., Sosin, C., Rich, R. M., \& Meylan, G. 1999, AJ, 118, 1727

Piotto, G., et al. 2002, A\&A, 391, 945

Rosenberg, A., Aparicio, A., Saviane, I., \& Piotto, G. 2000a, A\&AS, 145, 451 Rosenberg, A., Piotto, G., Saviane, I., \& Aparicio, A. 2000b, A\&AS, 144, 5

Rosenberg, A., Saviane, I., Piotto, G., \& Aparicio, A. 1999, AJ, 118, 2306

Rutledge, G. A., Hesser, J. E., \& Stetson, P. B. 1997, PASP, 109, 907

Sandage, A., \& Wildey, R. 1967, ApJ, 150, 469

Sarajedini, A., \& Layden, A. C. 1995, AJ, 109, 1086

Stetson, P. B. 1987, PASP, 99, 191

. 1994, PASP, 106, 250

Sweigart, A. V., Mengel, J. G., \& Demarque, P. 1974, A\&A, 30, 13

van den Bergh, S. 1967, AJ, 72, 70

Whitney, J. H., et al. 1998, ApJ, 495, 284 